\title{
Fungal mannosylation enhances human papillomavirus 16 E7 therapeutic immunity against TC-1 tumors
}

\author{
ZONGLIN WANG $^{1,2}$, CUIHUA WEI $^{1,2}$, YANJUN ZHANG $^{1,2}$, WEI WANG $^{1}$, ZHENG ZHOU $^{1}$ and GENGFU XIAO ${ }^{1}$ \\ ${ }^{1}$ State Key Laboratory of Virology, Wuhan Institute of Virology, Chinese Academy of Sciences, Wuhan, \\ Hubei 430071; ${ }^{2}$ University of the Chinese Academy of Sciences, Beijing 100039, P.R. China
}

Received May 9, 2017; Accepted October 27, 2017

DOI: 10.3892/or.2017.6083

\begin{abstract}
Cervical cancer, resulting from infection with human papillomavirus (HPV)16, remains the fourth most common cancer in women worldwide. Recently, three prophylactic HPV vaccines targeting high-risk HPVs (particularly HPV16 and HPV18) have been implemented to protect younger women. However, individuals with pre-existing infections have no benefit from prophylactic vaccines. Thus, there is an urgent need to develop therapeutic vaccines. HPV16 E7 has been widely utilized as a target for immune therapy of HPV16-associated lesions or cancers, reflecting the sustained existence of this virus in cancerous cells. We developed mannosylated HPV16 E7 (mE7) expressed from Pichia pastoris as a therapeutic vaccine against HPV16-associated cancer. Unmannosylated E7 (E7) was also generated from Pichia pastoris as a control. Mannosylation enhanced the uptake of mE7 by mannose receptors of bone marrow-derived dendritic cells (BMDCs), while the uptake of E7 was unaffected. mE7-uptake BMDCs in vitro induced more IFN- $\gamma$ secretion by splenocytes of immunized mice than E7. Vaccination of C57BL/6 mice with mE7 combined with adjuvant monophosphoryl lipid A (MPL) elicited stronger Th1 (type $1 \mathrm{~T}$ helper cell) responses and E7-specific $\mathrm{T}$ cell responses than E7. The $\mathrm{mE} 7$ vaccine induced the increased production of IFN- $\gamma$, IL-2 and TNF- $\alpha$, elicited more E7-specific IFN- $\gamma$-secreting $\mathrm{CD}^{+} \mathrm{T}$ cells in spleen and peripheral blood mononuclear cells (PMBCs)
\end{abstract}

Correspondence to: Dr Gengfu Xiao, State Key Laboratory of Virology, Wuhan Institute of Virology, Chinese Academy of Sciences, Wuhan, Hubei 430071, P.R. China

E-mail: xiaogf@wh.iov.cn

Abbreviations: HPV, human papillomavirus; BMDCs, bone marrow-derived dendritic cells; Th1, type 1 T helper cells; MPL, monophosphoryl lipid A; mE7, mannosylated HPV16 E7; DCs, dendritic cells; MRs, mannose receptors; PBMCs, peripheral blood mononuclear cells; PMA, phorbol-12-myristate-13-acetate

Key words: human papillomavirus 16, mannosylation, E7, therapeutic vaccine, TC-1 tumors and promoted stronger E7-specific cytotoxic $\mathrm{CD}^{+} \mathrm{T}$ cell responses compared with E7. Furthermore, TC-1 tumor challenged mice were used to confirm the antitumor activity of the vaccines. As a result, mE7 generated complete antitumor activity against TC-1 tumors, while E7 only provided partial antitumor activity. Taken together, mE7 can be a promising immunotherapy for treating cervical cancer.

\section{Introduction}

Cervical cancer is the fourth most common cancer in women, with an estimated 527,600 new cases and 265,700 deaths worldwide (1). Infection with high-risk types of human papillomavirus (HPV) leads to nearly all types of cervical cancers. HPV16 is the predominant HPV, causing $47.7 \%$ of cervical cancer cases in sub-Saharan Africa to $69.7 \%$ of cervical cancer cases in Europe/North America (2). In addition to cervical cancer, HPV infection is also associated with other anogenital and oral cavity cancers in the penis, vulva/vagina, anus, mouth, and oropharynx (3). To date, some prophylactic HPV vaccines have been administered to young women prior to the age of onset of sexual activity to protect against high-risk HPV infections $(4,5)$. Although these prophylactic vaccines have made great success in protecting younger women, individuals with pre-existing infections have no benefit from prophylactic vaccines (6). Thus, the research and development of therapeutic HPV16 vaccines are intensely required (7).

E6 and E7, two oncogenic HPV16 proteins, are required for the transformation of infected cells and maintenance of the transformed state $(8,9)$. Cervical cancers continuously express E6 and E7 as a result of the selective integration of the HPV16 genome into transformed cells (9). Thus, E6 and E7 represent promising targets for therapeutic HPV16 vaccines. Accordingly, numerous therapeutic HPV16 vaccines that elicit strong anti-E6/E7 cellular immunity or tumor regression have been reported, including peptide immunization, DNA immunization, E6/E7 fusion protein immunization, immunization with recombinant E7-expressing vaccinia virus, and E7-pulsed dendritic cells (7).

Dendritic cells (DCs) play a central role in the induction of adaptive immune responses (10). An effective manner of improving vaccination is targeting the receptor-mediated endocytosis of DCs. Herein, mannose receptors (MRs), the 
C-type lectins containing mannose receptor (MR, CD206), DC-SIGN (CD209), dectin-1 and langerin, have a high affinity for mannosylated antigens $(11,12)$. The uptake of mannosylated antigens by these C-type lectins has great advantages over other approaches (e.g., pinocytosis) in antigen presentation and $\mathrm{T}$ cell stimulation (13-16). Based on these advantages, antigen mannosylation represents a promising strategy for therapeutic vaccines in inducing cell-mediated immune responses $(17,18)$.

As previously reported, the mannosylation of the HPV16 E7 peptide enhanced the proliferation and activation of antigen-specific $\mathrm{CD} 8^{+} \mathrm{T}$ cells and the cytotoxic $\mathrm{T}$ cell response and increased antitumor activity in mice $(19,20)$. In the present study, we exploited Pichia pastoris to generate recombinant mannosylated HPV16 E7, which efficiently stimulated Th1 (type $1 \mathrm{~T}$ helper cell) and cell-mediated immune responses in the presence of monophosphoryl lipid A (MPL). In addition, mannosylation enhanced the uptake of mE7 by MRs of peripheral blood mononuclear cells (BMDCs) which then in vitro stimulated IFN- $\gamma$ secretion by splenocytes of immunized mice. Compared with E7, vaccination with mE7 combined with MPL of C57BL/6 mice increased the production of cytokines (IFN- $\gamma$, IL-2 and TNF- $\alpha$ ), induced more E7-specific IFN- $\gamma$ secreting $\mathrm{CD}^{+}{ }^{+} \mathrm{T}$ cells in the spleen and PMBCs, promoted E7-specific cytotoxic CD8 ${ }^{+} \mathrm{T}$ cell responses, and improved antitumor effects against HPV16 E7-expressing tumors. Hence, recombinant $\mathrm{mE} 7$ provides a promising immunotherapy for treating cervical cancer.

\section{Materials and methods}

Cells and culture. TC-1 cells, generated by the co-transformation of C57BL/6 mouse lung epithelial cells with HPV16 $E 6$ and $E 7$ oncogenes and the human ras oncogene (21), were purchased from the Cancer Hospital, Chinese Academy of Medical Sciences (ATCC no. CRL-2785). TC-1 cells were cultured in RPMI-1640 medium (HyClone, Logan, UT, USA), containing 10\% fetal bovine serum (FBS; Gibco, Grand Island, NY, USA), $100 \mathrm{U} / \mathrm{ml}$ penicillin, and $100 \mathrm{U} / \mathrm{ml}$ streptomycin (Beyotime, Shanghai, China). R10 medium, used for culturing BMDCs, contains standard RPMI-1640 plus $50 \mathrm{nM} \beta$-mercaptoethanol (Biosharp, Hefei, China). All cells were cultured at $37^{\circ} \mathrm{C}$ in humidified air supplemented with $5 \% \mathrm{CO}_{2}$.

Mice. Specific pathogen-free (SPF) 6- to -8-week-old female C57BL/6 mice were maintained at the Laboratory Animal Center of Wuhan Institute of Virology, Chinese Academy of Sciences (CAS). All mouse studies were performed according to Regulations of the Administration of Affairs Concerning Experimental Animals in China (WIVA25201304), and the protocols were reviewed and approved by the Laboratory Animal Care and Use Committee at the Wuhan Institute of Virology, CAS. Briefly, all the mice were fed in an independent ventilated cage (IVC) and the IVCs were kept within an experimental animal barrier environment. The food was sterilized by $\mathrm{Co}^{60}$-irradiation and the water was sterilized using an autoclave. We replenished the food and water twice or three times a week.
Cloning, expression and purification of $m E 7$ and $E 7$ in Pichia pastoris KM71. mE7 was generated from the oncogenic E7 gene of HPV16 (GenBank accession no. AF125673) synthesized at Sangon Biotech (Shanghai, China) and a linker encoding 15-amino acids containing two N-X-S/T motifs (GGGGSNGTGGGNASC) with two potential $N$-linked glycosylation sites. In contrast to mE7, E7 was generated from the site-directed mutagenesis of the two potential $N$-linked glycosylation sites in the linker region. NGT and NAS were conservatively mutated to AGT and AAS, respectively.

The mE7 and E7 were cloned into plasmid pPICZ $\alpha \mathrm{A}$. Subsequently, recombinant plasmids pPICZ $\alpha \mathrm{A}-\mathrm{mE} 7$ and pPICZ $\alpha$ A-E7 were linearized using SacI (NEB, Ipswich, MA, USA), purified, transfected into electrocompetent Pichia pastoris KM71 (pulsed $1.5 \mathrm{kV}, 200 \Omega$, and $25 \mu \mathrm{F}$ ) and subsequently incubated for $2 \mathrm{~h}$ in yeast extract peptone dextrose medium (YPD). Recombinant clones were selected on YPD agar plates, containing $75 \mu \mathrm{g} / \mathrm{ml}$ Zeocin (Invitrogen, San Diego, CA, USA), and identified using colony PCR. The expression and purification of recombinant protein in KM71 was conducted according to Lam et al (15).

Generation of BMDCs. BMDCs were generated according to Lutz et al (22). Briefly, bone marrow cells from the tibiae and femurs of one 8-week-old C57BL/6 mouse were cultured in R10 medium supplemented with $200 \mathrm{U} / \mathrm{ml} \mathrm{rmGM-CSF}$ (PeproTech, Rocky Hill, NJ, USA). The cells were fed with fresh medium on days 3,6 , and 8 and matured with fresh R10 medium containing $100 \mathrm{U} / \mathrm{ml} \mathrm{rmGM-CSF}$ and $500 \mathrm{U} / \mathrm{ml}$ TNF- $\alpha$ (PeproTech) on day 10.

BMDCs uptake of recombinant protein and $T$ cell polarization. The mature BMDCs were cultured in $6-\mathrm{cm}$ plates and co-incubated with $10 \mu \mathrm{g} / \mathrm{ml} \mathrm{mE7} \mathrm{or} \mathrm{E7} \mathrm{in} \mathrm{the} \mathrm{presence} \mathrm{or}$ absence of $10 \mathrm{mg} / \mathrm{ml}$ yeast mannans (Sigma, St. Louis, MO, USA) for $45 \mathrm{~min}$. Subsequently, the cells were collected and washed using PBS. The collected cells were lysed using RIPA lysis buffer (Beyotime), and centrifuged at 10,000 $\mathrm{x}$ g for $5 \mathrm{~min}$ to remove the debris. The supernatants were precipitated with 4 volumes of acetone, incubated at $-20^{\circ} \mathrm{C}$ overnight, centrifuged at $13,000 \mathrm{x}$ g for $15 \mathrm{~min}$ and subsequently analyzed by western blotting.

$\mathrm{T}$ cell polarization assays were performed following the method of Satchidanandam et al (23). Briefly, the mE7- or E7-preincubated BMDCs were cultured for $24 \mathrm{~h}$ and then treated with $50 \mu \mathrm{g} / \mathrm{ml}$ mitomycin C (Sigma) for $1 \mathrm{~h}$ followed by extensive washing. The above BMDCs were co-cultured in a 96-well plate with one million splenocytes from C57BL/6 mice immunized with mE7, E7 or PBS, at the BMDC:splenocyte ratio of 1:10 for $72 \mathrm{~h}$. The supernatants were collected for IFN- $\gamma$ measurement using capture ELISA (BioLegend, San Diego, CA, USA), according to the protocol for Mouse IFN- $\gamma$ ELISA MAX Standard Sets.

Vaccination of mice with recombinant proteins. The 6- to 8-week-old C57BL/6 mice were grouped and subcutaneously (s.c.) vaccinated with $40 \mu \mathrm{g} \mathrm{mE7,} 40 \mu \mathrm{g}$ E7 or $100 \mu \mathrm{l} \mathrm{PBS}$ in the presence of $5 \mu \mathrm{g}$ adjuvant MPL (Sigma, L6895) on days 0 , 5 and 10. On day 15, the mice were sacrificed by cervical dislocation under aseptic conditions. Eyeball enucleation was 

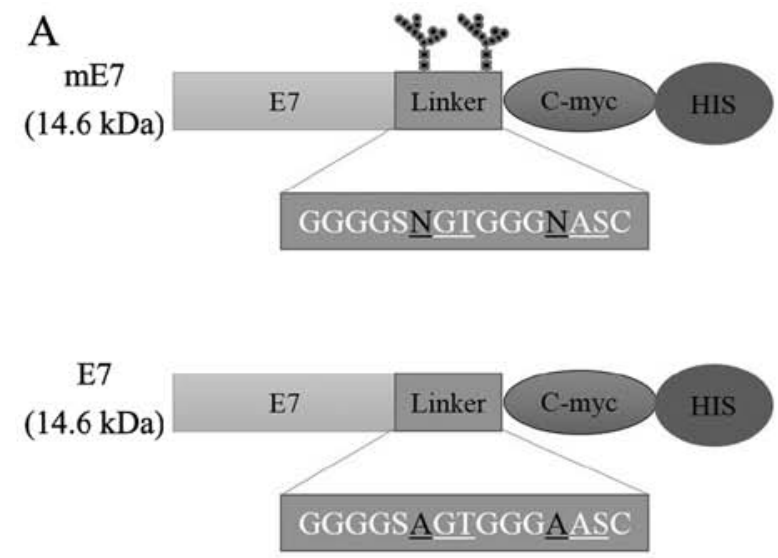

N-linked mannosylation
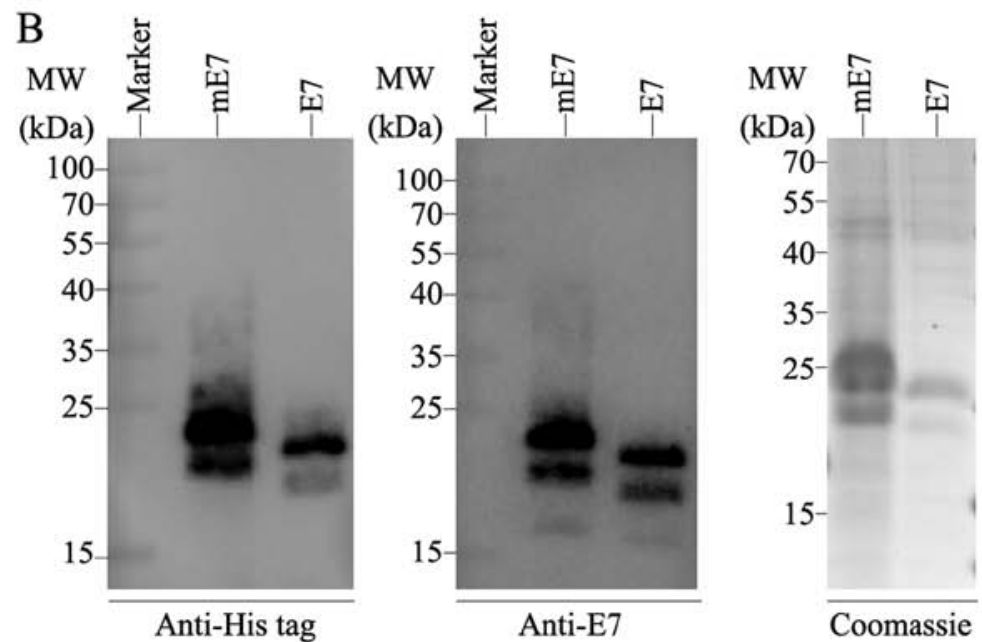

Figure 1. Construction, expression and purification of mE7 and E7 in KM71. (A) Schematic representation of mE7 and E7. All proteins comprised HPV16 E7, a 15-amino acid linker, the c-Myc epitope and a $6 \mathrm{X}$ His tag. The linker region of mE7 contains two potential $N$-linked glycosylation sites: NGT and NAS, which were mutated to AGT and AAS (E7). (B) Western blotting and Coomassie blue staining analysis of purified mE7 and E7. The samples were analyzed using $15 \%$ SDS-PAGE, followed by western blotting and Coomassie blue staining. Mouse mAbs anti-His tag and anti-E7 were used to detect the purified mE7 and E7, with a molecular weight between 22 and $40 \mathrm{kDa}$.

conducted for blood collection. The sera and splenocytes were collected and harvested from blood.

Cytokine quantification. Four million splenocytes from each immunized mouse were cultured for $48 \mathrm{~h}$ in 24-well plates in the presence of $10 \mu \mathrm{g} / \mathrm{ml} \mathrm{E} 7$ or phorbol-12-myristate13-acetate (PMA) plus ionomycin (Dakewe Biotech Co., Shenzhen, China). The supernatants were collected, and the concentrations of IFN- $\gamma$, IL- 2 and TNF- $\alpha$ were measured using capture ELISA (BioLegend), according to the protocols for Mouse IFN- $\gamma /$ IL-2/TNF- $\alpha$ ELISA MAX Standard Sets.

Antibody determination. The sera were collected from each immunized mouse at 5 days after the last immunization to determine specific IgG using ELISA. Polyvinylchloride 96-well plates (Nunc, Rochester, NY, USA) were coated with E7 at $2 \mu \mathrm{g} / \mathrm{ml}$ in PBS and incubated overnight at $4^{\circ} \mathrm{C}$. The plates were subsequently blocked with $10 \%$ FBS in PBS, washed, and incubated with the two-fold diluted serums, followed by detection with HRP-conjugated Affinipure Goat Anti-Mouse IgG (Proteintech, Wuhan, China).

FACS staining. One million splenocytes from each immunized mouse and PBMCs from each group were cultured for $24 \mathrm{~h}$ in 96 -well plates in the presence of $10 \mu \mathrm{g} / \mathrm{ml}$ E7 or PMA plus ionomycin as positive control. For surface staining, cells were incubated with the following antibodies (BD Biosciences, Franklin Lakes, NJ, USA): PE-Cy7 anti-CD3e (145-2C11), FITC anti-CD4 (RM4-5), PE anti-CD8a (53-6.7) and 7-AAD Viability Staining Solution (BioLegend). For intracellular IFN- $\gamma$ staining, APC anti-IFN- $\gamma$ (XMG1.2, BD Biosciences) was used. All the staining procedures were according to BD Biosciences protocols. Data were acquired using a BD FACS flow cytometer and analyzed by FlowJo software (Tree Star, Ashland, OR, USA).
Cytotoxicity assays. Sixteen million splenocytes from each immunized mouse were cocultured with one million mitomycin C-pretreated TC-1 cells in RPMI-1640 supplemented with $10 \%$ FBS, $50 \mu \mathrm{g} / \mathrm{ml}$ concanavalin A (Con A; Sigma) and $20 \mathrm{U} / \mathrm{ml} \mathrm{IL-2} \mathrm{(PeproTech)} \mathrm{at} 37^{\circ} \mathrm{C}$ in $5 \% \mathrm{CO}_{2}$. After 5 days, the viable splenocytes were collected and used as effector cells, and TC-1 cells were used as target cells. The Pierce LDH Cytotoxicity assay kit (Thermo Fisher Scientific, Waltham, MA, USA) was used to measure the effector cells against TC-1 at ratios of 2.5:1 and 5:1 according to the manufacturer's instructions. Specific lysis was calculated as percent specific lysis $=[($ Experimental value - Effector cell spontaneous control - Target cell spontaneous control)/ (Target cell maximum control - Target cell spontaneous control)] x100.

Tumor challenge. On day 0, the 6- to 8-week-old C57BL/6 mice were injected (s.c.) with $2 \times 10^{5}$ TC-1 cells in the right flank. Vaccination with $\mathrm{mE7}, \mathrm{E} 7$ and PBS with MPL was performed on days 3,8, and 13. Tumor growth was measured using a caliper, and tumor size was calculated as volume $=\left(\right.$ length $\mathrm{x}$ width $\left.{ }^{2}\right) / 2(24)$. For survival analysis, the mice were considered as dead when the tumor reached $1000 \mathrm{~mm}^{3}$. When the tumor size of the immunized mice exceeded $1000 \mathrm{~mm}^{3}$, the mice were sacrificed and dissected. Tumors were separated by ophthalmic scissors and tweezers, and kept in formalin. Tumor-free mice vaccinated with mE7 were challenged with $2 \times 10^{5}$ TC- 1 cells again on day 31 . The percentage of tumor-free mice was recorded.

Statistical analysis. Statistical analysis was performed using Prism software version 6.0 (GraphPad, San Diego, CA, USA). All data are presented as the means \pm SD. The cytokine concentration, antibody titers and FACS results were assessed using an unpaired two-tailed t-test. Cytotoxicity assays and tumor sizes were assessed using two-way ANOVA multiple comparisons. Survival percentages were assessed using the 
A
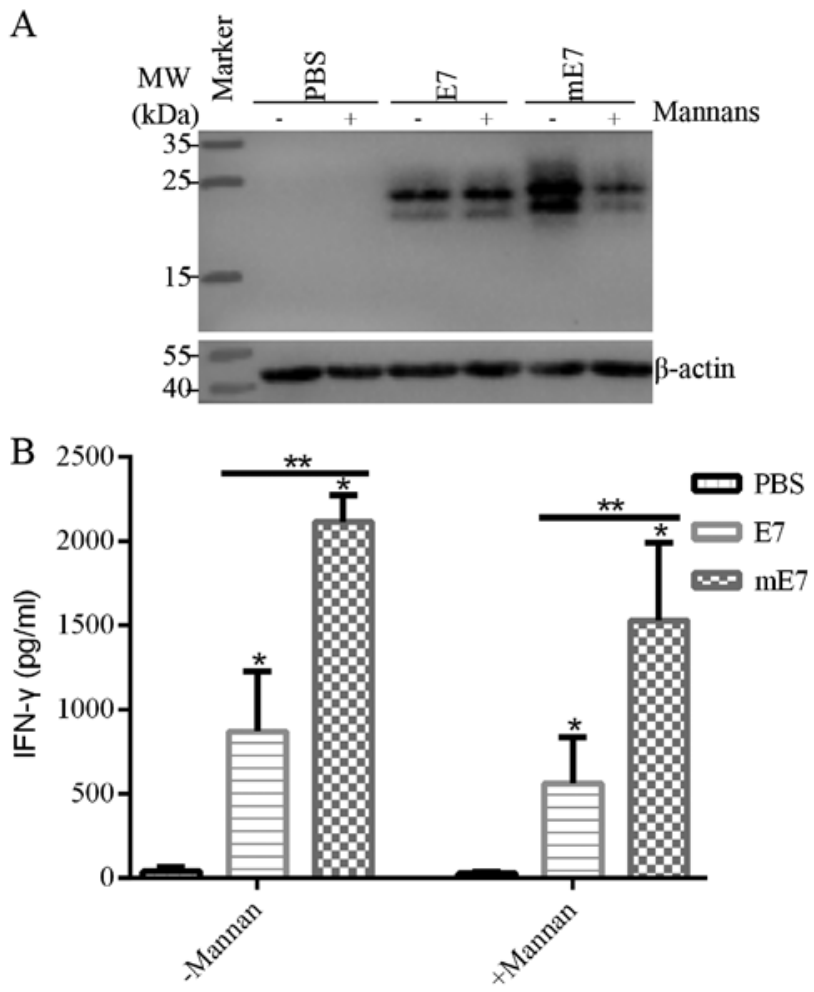

Figure 2. Mannosylation enhances uptake of mE7 by BMDCs and T cell polarization. BMDCs were incubated with $10 \mu \mathrm{g} / \mathrm{ml} \mathrm{mE7} \mathrm{or} \mathrm{E7} \mathrm{in} \mathrm{the}$ absence (-) or presence (+) of $10 \mathrm{mg} / \mathrm{ml}$ mannans for $45 \mathrm{~min}$. (A) BMDCs were lysed and subjected to western blotting. Mouse $\mathrm{mAbs}$ anti-E7 and anti$\beta$-actin were used to detect intracellular mE7 or E7 and $\beta$-actin. (B) T cell polarization. Antigen-uptake BMDCs were cultured for another $24 \mathrm{~h}$, then killed by mitomycin $\mathrm{C}$, and co-cultured with splenocytes of immunized mice for $72 \mathrm{~h}$. IFN- $\gamma$ of the supernatants was measured by ELISA. Each group comprised 3 mice. The values marked with asterisks in this figure: ${ }^{*} \mathrm{P}<0.05$ ${ }^{* *} \mathrm{P}<0.01$ (comparing PBS with mE7 or E7, and comparing mE7 with E7).

log-rank test. P-values of $<0.05$ were considered to indicate statistically significant differences.

\section{Results}

Construction and expression of $m E 7$ and E7. As previously reported, Pichia pastoris has been widely used to express mannosylated recombinant proteins (25). To express a mannosylated recombinant E7 (defined as mE7), a 15-amino acid linker containing two N-X-S/T motifs representing potential $N$-linked glycosylation sites was fused to the C-terminal of HPV16 E7. As a control, E7 was subjected to the site-directed mutagenesis of the two potential $N$-linked glycosylation sites in the linker region of mE7. NGT and NAS were conservatively mutated to AGT and AAS, respectively (Fig. 1A).

The mE7 and E7 recombinant proteins were efficiently expressed by Pichia pastoris KM71 in secreted forms, and mE7 showed a molecular weight between 22 and $40 \mathrm{kDa}$, while E7 showed a molecular weight of $\sim 22 \mathrm{kDa}$ (Fig. 1B), indicating the considerable glycosylation of mE7 in KM71 and the unglycosylation of E7, since the unglycosylated E7 expressed from BL21 (DE3) cells was $22 \mathrm{kDa}$ (data not shown). The optimum time for mE7 expression $(72 \mathrm{~h}$ ) was longer than that for E7 (36 h).
Table I. Relative quantification of bands in the different lanes (Fig. 2A).

\begin{tabular}{lcccccc}
\hline & PBS & PBS+M & E7 & E7+M & $\mathrm{mE7}$ & $\mathrm{mE7}+\mathrm{M}$ \\
\hline$\beta$-actin & 1.0 & 0.76 & 1.03 & 1.08 & 0.89 & 1.05 \\
Antigen & 0 & 0 & 1.42 & 1.35 & 2.00 & 1.07 \\
Antigen/\$-actin & 0 & 0 & 1.38 & 1.25 & 2.25 & 1.02 \\
\hline
\end{tabular}

All antigens contain two portions in the same lane. $M$ in the above table represents mannans. The band area and gray value of different lanes were normalized when the values of $\beta$-actin and PBS were set as 1.0 , respectively.

Mannosylation enhances uptake of $m E 7$ by the MRs of $B M D C s$ and $T$ cell polarization. To detect whether mannosylated proteins are more efficiently taken up by DCs via MR-mediated endocytosis, BMDCs were incubated with equal $\mathrm{mE7}$ or E7 in the presence or absence of yeast mannans, which block MR and DC-SIGN (26). BMDCs endocytosed more $\mathrm{mE7}$ than E7, and yeast mannans inhibited the uptake of mE7, while E7 was not apparently affected (Fig. 2A). As shown in Table I, the relative amount of mE7 ranged from 2.25 (without mannans) to 1.02 (with mannans) while that of E7 ranged from 1.38 (without mannans) to 1.25 (with mannans). This result indicates that MR or DC-SIGN plays a dominant role in the uptake of mannosylated proteins by BMDCs, and mannosylation can promote the uptake of $\mathrm{mE7}$ by BMDCs, which is crucial to activate downstream immune responses. Subsequently, we co-cultured antigen-uptake BMDCs with splenocytes of immunized mice for $72 \mathrm{~h}$. As a results, mE7-uptake BMDCs induced more IFN- $\gamma$ secretion by splenocytes than E7, while the levels of IFN- $\gamma$ were reduced with mannans, respectively (Fig. 2B). Taken together, mannosylation enhances the ability of BMDCs to take up mE7 and increases $\mathrm{T}$ cell polarization.

Vaccination with $m E 7$ induces strong Th1 responses. We measured the E7-specific antibody titers in the sera of immunized mice using ELISA. The results showed that vaccination with mE7 developed higher antibody titer than E7 (Fig. 3A).

To assess the level of cytokines generated by vaccination with $\mathrm{mE7}$, the splenocytes from vaccinated mice were harvested and stimulated with E7 or a positive control PMA plus ionomycin. Subsequently, the supernatants were collected and measured using ELISA. Under equal doses of mE7 and E7, vaccination with $\mathrm{mE7}$ induced more cytokines, including IFN- $\gamma$, IL-2 and TNF- $\alpha$, than vaccination with E7 (Fig. 3B-D). Compared with E7, mE7 induced almost six times more IFN- $\gamma$ (Fig. 3B), which is an important activator of macrophages, inducer of MHC II expression and indicator of Th1 responses. Compared with E7, mE7 also induced approximately twice as much IL-2 (Fig. 3C), which stimulates the differentiation of regulatory $\mathrm{T}$ cells, promotes $\mathrm{T}$ cell growth and NK cytolytic activity, and mediates activationinduced cell death (27). In addition, compared with E7, mE7 induced approximately twice as much TNF- $\alpha$ (Fig. 3D), which induces fever, apoptotic cell death, cachexia, inflammation and inhibition of tumorigenesis. Moreover, the E7-specific 

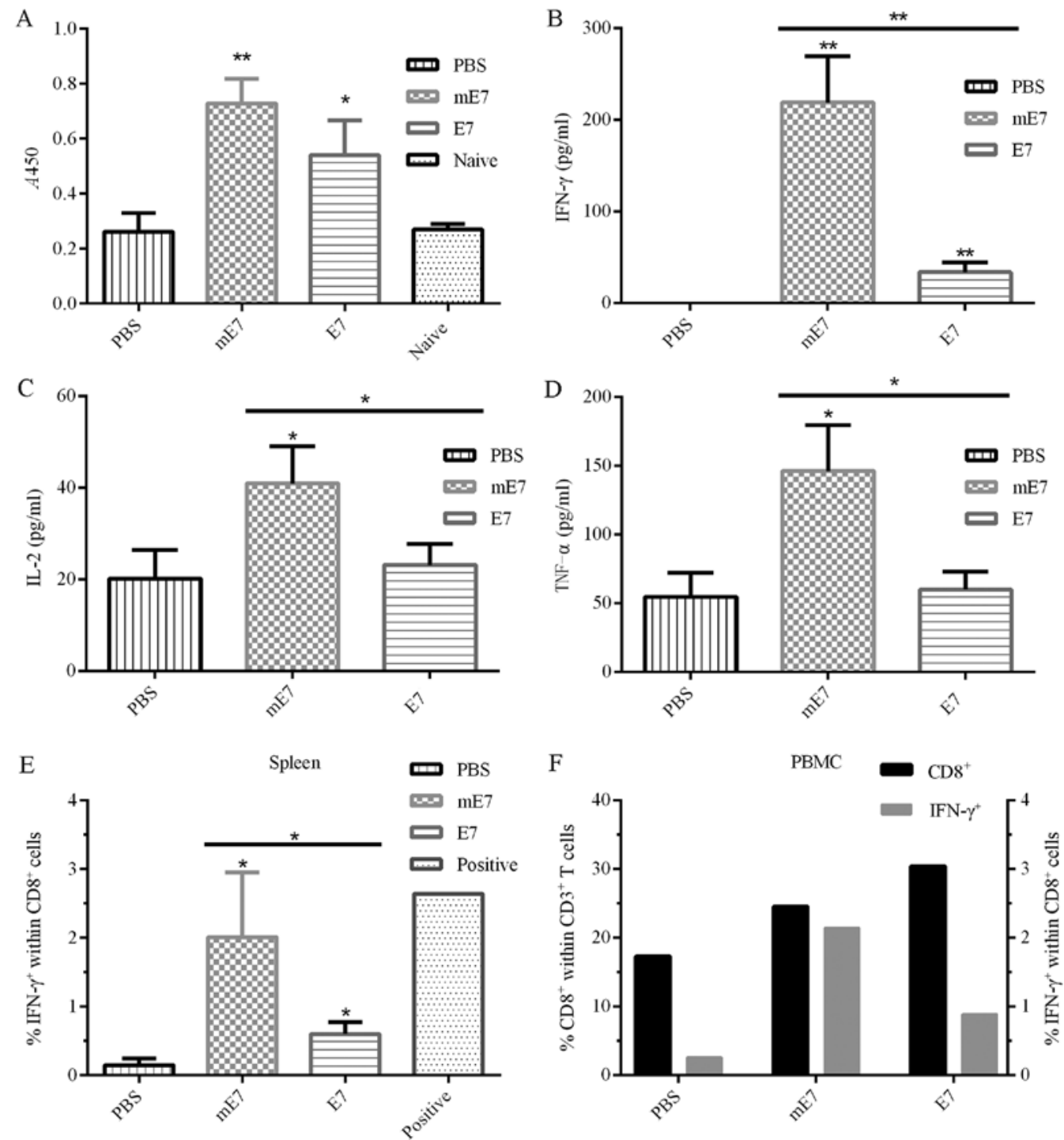

Figure 3. Vaccination with $\mathrm{mE7}$ induces strong Th1 responses. (A) E7-specific antibody determination. The sera of vaccinated mice were collected and subjected to ELISA to determine E7-specific antibody titers. The results show a dilution of 1:640. Cytokine quantification of IFN- $\gamma$ (B), IL-2 (C) and TNF- $\alpha$ (D). The mice were immunized with $\mathrm{mE7}$, E7 or PBS on days 0,5,10 and sacrificed on day 15 . The splenocytes and PBMCs of the vaccinated mice were harvested and co-cultured with E7 for $48 \mathrm{~h}$. The supernatants were evaluated via ELISA for IFN- $\gamma$, IL-2 and TNF- $\alpha$ quantification. Analysis of E7-specific IFN- $\gamma$ secreting $\mathrm{CD} 8^{+} \mathrm{T}$ cell within the spleen (E) and PBMCs (F) with flow cytometry. The above splenocytes and PBMCs were stimulated with E7 for $24 \mathrm{~h}$, and then stained for CD8 and intracellular IFN- $\gamma$. The values marked with asterisks in these figures: ${ }^{*} \mathrm{P}<0.05 ;{ }^{* * *} \mathrm{P}<0.01$ (comparing PBS with $\mathrm{mE} 7$ or E7, and comparing mE7 with E7). Each group comprised 3 mice.

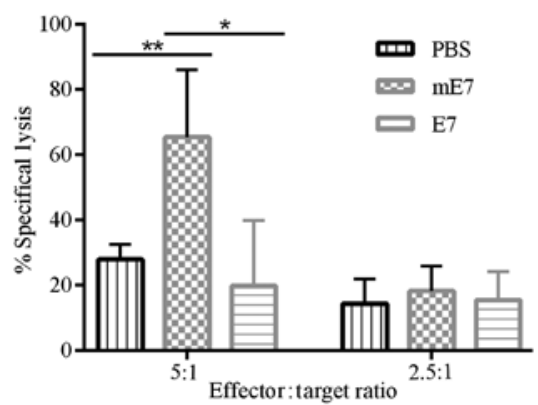

Figure 4. Vaccination with $\mathrm{mE} 7$ promotes E7-specific cytotoxic $\mathrm{CD} 8^{+} \mathrm{T}$ cell response. The mice were immunized with $\mathrm{mE7}$, E7 or PBS on days 0,5 , and 10 and sacrificed on day 15 . The splenocytes of vaccinated mice were harvested and cocultured in vitro with mitomycin C-treated TC-1 cells. E7-specific cytolytic activity was assayed against TC-1 cells. The values marked with asterisks in this figure: ${ }^{*} \mathrm{P}<0.05 ;{ }^{* *} \mathrm{P}<0.01$ (comparing $\mathrm{mE} 7$ with E7, and comparing PBS with mE7). Each group comprised 3 mice.
IFN- $\gamma$-secreting $\mathrm{CD} 8^{+} \mathrm{T}$ cells in the spleen and PBMCs were analyzed by flow cytometry (Fig. 3E and F). Compared with E7, mE7 induced more E7-specific IFN- $\gamma$-secreting CD8 ${ }^{+}$ $\mathrm{T}$ cell within the spleen and PBMCs. These results suggested that vaccination with $\mathrm{mE} 7$ generated stronger Th1 responses which are crucial for tumor regression and considerable E7-specific antibody responses.

Vaccination with $m E 7$ promotes $E 7$-specific cytotoxic $C D 8^{+}$ $T$ cell response. The above results that vaccination with mE7 promoted Th1 responses (Fig. 3B-F) represents potent E7-specific $\mathrm{T}$ cell responses. To examine whether vaccination with $\mathrm{mE} 7$ primes more effective E7-specific cytotoxic $\mathrm{CD} 8^{+} \mathrm{T}$ cells than $\mathrm{E} 7$, the splenocytes of vaccinated mice were collected and cocultured with mitomycin C-pretreated TC-1 cells for 5 days. Viable effector cells were measured for cytotoxic activity against TC-1 cells. As shown in Fig. 4, 

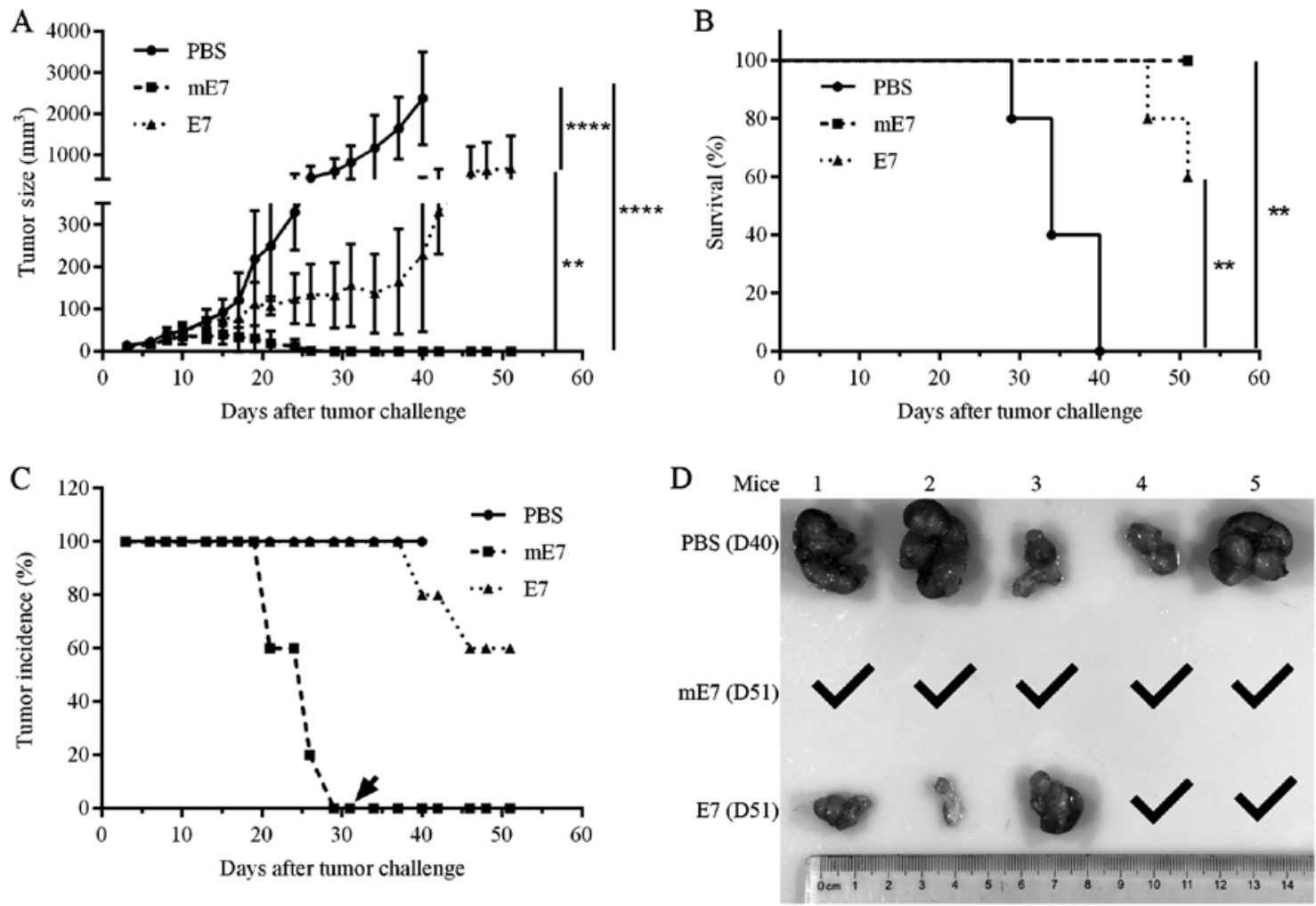

Figure 5. Mannosylation enhances mE7 antitumor activity against TC-1 tumors. C57BL/6 mice were injected with $2 \times 10^{5} \mathrm{TC}-1$ cells in the right flank on day 0 . Vaccination with $\mathrm{mE7}$, E7, and PBS (5 per group) in combination with MPL was performed on days 3, 8, and 13. (A) The tumor sizes were monitored for 51 days. Mice vaccinated with PBS were sacrificed when all the tumor sizes reached $1000 \mathrm{~mm}^{3}$. (B) Survival analysis of the vaccinated mice. (C) The percentage of tumor incidence was recorded. Black arrow in this figure: mice vaccinated with mE7 were re-challenged with $2 \times 10^{5} \mathrm{TC}-1$ cells on day 31 . (D) The tumors of the challenged mice were removed on days 40 and 51. The countermark in the image represents tumor regression. Values marked with asterisks in these figures: ${ }^{* *} \mathrm{P}<0.01 ;{ }^{* * * * *} \mathrm{P}<0.0001$ (comparing mE7 with E7, and comparing PBS with mE7 and E7). Each group comprised 5 mice. The experiments were repeated twice with similar results.

the effector cells from mice immunized with mE7 showed stronger cytolytic effects on TC-1 cells $(65.4 \%)$ than those from mice immunized with E7 (19.8\%) and PBS (27.9\%) when the effector:target ratio was 5:1.

Mannosylation enhances $m E 7$ antitumor activity against TC-1 tumors. To assess the antitumor activity generated using the $\mathrm{mE} 7$ vaccine, we performed an in vivo tumor challenge assay with HPV16 E6 and E7-expressing TC-1 cells. C57BL/6 mice were injected with $2 \times 10^{5} \mathrm{TC}-1$ cells in the right flank. After 3 days, tumor-challenged mice (5 per group) were vaccinated with three doses of $\mathrm{mE7}$, E7 or PBS in the presence of MPL every 5 days. Tumor growth was measured using a caliper every 2 or 3 days. As shown in Fig. 5A, the mice vaccinated with $\mathrm{mE7}$ showed lower average tumor sizes than mice vaccinated with E7 and PBS. All mice vaccinated with $\mathrm{mE7}$ eliminated tumors at 26 days after tumor challenge, while only $20 \%$ of the mice vaccinated with E7 eliminated tumors at more than 37 days after tumor challenge. All mice vaccinated with PBS failed to inhibit tumor growth and were sacrificed after 40 days, when the tumor sizes reached $1000 \mathrm{~mm}^{3}$. Additionally, all mice vaccinated with $\mathrm{mE} 7$ survived after 51 days. In contrast, $40 \%$ of the mice vaccinated with E7 and 100\% of the mice vaccinated with PBS died after 51 days (Fig. 5B and C). After 31 days, the mice vaccinated with $\mathrm{mE} 7$ were rechallenged with $2 \times 10^{5} \mathrm{TC}-1$ cells, and no mice developed tumors until 51 days (Fig. 5C), indicating immunological memory. At 40 and 51 days, the mice vaccinated with PBS, mE7 and E7 were sacrificed and dissected, and 5 PBS tumors and 3 E7 tumors were observed (Fig. 5D). Taken together, mE7 represents an effective therapeutic vaccine against TC-1 tumors.

\section{Discussion}

In the present study, we reported that mE7, expressed by Pichia pastoris, is an effective therapeutic vaccine candidate against HPV16 E7-expressing tumors. Mannosylation enhanced the uptake of E7 in BMDCs by MRs and T cell polarization. As a result, the vaccination of $\mathrm{C} 57 \mathrm{BL} / 6$ mice with $\mathrm{mE} 7$ induced more cytokines (IFN- $\gamma$, IL-2 and TNF- $\alpha$ ), more E7-specific IFN- $\gamma$-secreting CD8 ${ }^{+} \mathrm{T}$ cells in the spleen and PBMCs, and more E7-specific cytotoxic $\mathrm{CD} 8^{+} \mathrm{T}$ cell responses, suggesting the activation of E7-specific $\mathrm{T}$ cell responses. In tumor challenge assays, immunization with mE7 significantly inhibited tumor growth and prolonged the lifespan of tumor-challenged mice.

HPV16 E6 and E7 are often the only viral genes expressed in cancerous cells. Thus, E6 and E7 represent promising targets for the immune therapy of HPV-associated lesions or cancers. Therefore, numerous therapeutic vaccines have focused on E6 and E7, pursuing E6/E7-specific cellular immunity or tumor regression. Traditionally, vaccinations with peptide(s), including minimal or maximal epitopes, 
have been considered a convenient method to elicit cellular immunity. However, some limitations remain, as peptide vaccines may result in $\mathrm{T}$ cell tolerance and become rapidly degraded (28). In contrast, immunization with deleted (29) or chemically synthesized (30) E7 protein induces T cell immunity in the presence of Quil A or CpG. In addition, the fusion of E7 proteins with heat shock proteins, virus-like particles, or listeriolysin, elicits E7-specific $\mathrm{CD}^{+} \mathrm{T}$ cell responses (7). Moreover, E6/E7-containing DNA and viral vector vaccines have been exploited to promote T cell immunity (7). However, the DNA and viral vector vaccines present risks for the integration of exogenous E6 or E7 genes into host cells. In contrast, protein vaccines have some advantages: i) exogenous proteins have no expected access to host cells for carcinogenic effects; and ii) exogenous proteins transiently exist and avoid persistently transforming host cells. Taken together, these facts indicate the promising application of E6 or E7 protein-based vaccines. Pichia pastoris has been used to produce therapeutic glycoproteins (25). Since glycosylation is of the high-mannose type, we exploited Pichia pastoris to produce mE7 to augment the immunogenicity of E7, as $\mathrm{mE7}$ utilizes the immune recognition of exposed mannoses as pathogen-associated molecular patterns.

MRs play an important role in the immune system and connect innate to adaptive immunity (31). Previous studies have shown that MRs participate in the uptake, processing and presentation of glycosylated antigens as the immune response to foreign pathogens (32). DC maturation occurs after mannosylated antigens bind to MRs and trigger the internalization of the mannosylated antigens. We observed that Pichia pastoris mannosylation enhanced the uptake of mE7 by the MRs of BMDCs and promoted $\mathrm{T}$ cell polarization. These data indicate the involvement of MR and DC-SIGN in the uptake of mE7, as the uptake activity was inhibited when yeast mannans were added.

Studies have reported that the mannosylation of antigens enhances MHC I- and MHC II-restricted antigen presentation and $\mathrm{T}$ cell stimulation $(13,14,17,33,34)$. Furthermore, the immunization of mice with mannosylated vaccines induced Th1 cytokine production and antigen-specific CTL responses $(17,35,36)$. In the present study, vaccination with mE7 improved the production of Th1 cytokines and the level of E7-specific IFN- $\gamma$-secreting $\mathrm{CD}^{+} \mathrm{T}$ cell in the spleen and PBMCs, such as IFN- $\gamma$, IL- 2 and TNF- $\alpha$, and enhanced E7-specific CTL responses.

In conclusion, mannosylated yeast-derived antigens more effectively induce antigen-specific $\mathrm{T}$ cell proliferation than their unmannosylated counterparts. In the present study, mE7 showed more potent induction of E7-specific and cytotoxic $\mathrm{T}$ cell responses and antitumor activity than $\mathrm{E} 7$, providing a promising immunotherapy for treating cervical cancer.

\section{Acknowledgements}

The authors would like to thank the technicians of the Laboratory Animal Center of Wuhan Institute of Virology and Public Technology Service Center of Wuhan Institute of Virology for providing technical assistance. The present study was supported by the National Key Research and Development Program of China (2016YFC1200400).

\section{References}

1. Torre LA, Bray F, Siegel RL, Ferlay J, Lortet-Tieulent J and Jemal A: Global cancer statistics, 2012. CA Cancer J Clin 65: 87-108, 2015.

2. Muñoz N, Bosch FX, Castellsagué X, Díaz M, de Sanjose S, Hammouda D, Shah KV and Meijer CJ: Against which human papillomavirus types shall we vaccinate and screen? The international perspective. Int J Cancer 111: 278-285, 2004.

3. Howley P, Schiller J and Lowy D: Papillomaviruses. In: Fields Virology. Knipe DM and Howley PM (eds). 6th edition. Lippincott Williams \& Wilkins, Philadelphia, PA, pp1662-1703, 2013.

4. Herrero R, González P and Markowitz LE: Present status of human papillomavirus vaccine development and implementation. Lancet Oncol 16: e206-e216, 2015.

5. Schiller JT and Müller M: Next generation prophylactic human papillomavirus vaccines. Lancet Oncol 16: e217-e225, 2015.

6. Hildesheim A, Herrero R, Wacholder S, Rodriguez AC, Solomon D, Bratti MC, Schiller JT, Gonzalez P, Dubin G, Porras C, et al; Costa Rican HPV Vaccine Trial Group: Effect of human papillomavirus 16/18 L1 viruslike particle vaccine among young women with preexisting infection: A randomized trial. JAMA 298: 743-753, 2007.

7. Su JH, Wu A, Scotney E, Ma B, Monie A, Hung CF and Wu TC: Immunotherapy for cervical cancer: Research status and clinical potential. BioDrugs 24: 109-129, 2010.

8. zur Hausen H: Papillomaviruses and cancer: From basic studies to clinical application. Nat Rev Cancer 2: 342-350, 2002.

9. Frazer IH: Prevention of cervical cancer through papillomavirus vaccination. Nat Rev Immunol 4: 46-54, 2004.

10. Mellman I and Steinman RM: Dendritic cells: Specialized and regulated antigen processing machines. Cell 106: 255-258, 2001.

11. Drickamer K: C-type lectin-like domains. Curr Opin Struct Biol 9: 585-590, 1999.

12. McGreal EP, Miller JL and Gordon S: Ligand recognition by antigen-presenting cell C-type lectin receptors. Curr Opin Immunol 17: 18-24, 2005.

13. Engering AJ, Cella M, Fluitsma D, Brockhaus M, Hoefsmit EC, Lanzavecchia A and Pieters J: The mannose receptor functions as a high capacity and broad specificity antigen receptor in human dendritic cells. Eur J Immunol 27: 2417-2425, 1997.

14. Tan MC, Mommaas AM, Drijfhout JW, Jordens R, Onderwater JJ, Verwoerd D, Mulder AA, van der Heiden AN, Scheidegger D, Oomen LC, et al: Mannose receptor-mediated uptake of antigens strongly enhances HLA class II-restricted antigen presentation by cultured dendritic cells. Eur J Immunol 27: 2426-2435, 1997.

15. Lam JS, Mansour MK, Specht CA and Levitz SM: A model vaccine exploiting fungal mannosylation to increase antigen immunogenicity. J Immunol 175: 7496-7503, 2005.

16. Levitz SM and Specht CA: The molecular basis for the immunogenicity of Cryptococcus neoformans mannoproteins. FEMS Yeast Res 6: 513-524, 2006.

17. Keler T, Ramakrishna V and Fanger MW: Mannose receptortargeted vaccines. Expert Opin Biol Ther 4: 1953-1962, 2004.

18. Irache JM, Salman HH, Gamazo C and Espuelas S: Mannosetargeted systems for the delivery of therapeutics. Expert Opin Drug Deliv 5: 703-724, 2008

19. Moyle PM, Olive C, Ho MF, Pandey M, Dyer J, Suhrbier A, Fujita Y and Toth I: Toward the development of prophylactic and therapeutic human papillomavirus type-16 lipopeptide vaccines. J Med Chem 50: 4721-4727, 2007.

20. Rauen J, Kreer C, Paillard A, van Duikeren S, Benckhuijsen WE, Camps MG, Valentijn AR, Ossendorp F, Drijfhout JW, Arens R, et al: Enhanced cross-presentation and improved $\mathrm{CD} 8^{+} \mathrm{T}$ cell responses after mannosylation of synthetic long peptides in mice. PLoS One 9: e103755, 2014.

21. Lin KY, Guarnieri FG, Staveley-O'Carroll KF, Levitsky HI, August JT, Pardoll DM and Wu TC: Treatment of established tumors with a novel vaccine that enhances major histocompatibility class II presentation of tumor antigen. Cancer Res 56: 21-26, 1996.

22. Lutz MB, Kukutsch N, Ogilvie AL, Rössner S, Koch F, Romani N and Schuler G: An advanced culture method for generating large quantities of highly pure dendritic cells from mouse bone marrow. J Immunol Methods 223: 77-92, 1999. 
23. Satchidanandam V, Kumar N, Jumani RS, Challu V, Elangovan S and Khan NA: The glycosylated Rv1860 protein of Mycobacterium tuberculosis inhibits dendritic cell mediated TH1 and TH17 polarization of T cells and abrogates protective immunity conferred by BCG. PLoS Pathog 10: e1004176, 2014.

24. Naito S, von Eschenbach AC, Giavazzi R and Fidler IJ: Growth and metastasis of tumor cells isolated from a human renal cell carcinoma implanted into different organs of nude mice. Cancer Res 46: 4109-4115, 1986.

25. Gerngross TU: Advances in the production of human therapeutic proteins in yeasts and filamentous fungi. Nat Biotechnol 22 : 1409-1414, 2004.

26. Sallusto F, Cella M, Danieli C and Lanzavecchia A: Dendritic cells use macropinocytosis and the mannose receptor to concentrate macromolecules in the major histocompatibility complex class II compartment: Downregulation by cytokines and bacterial products. J Exp Med 182: 389-400, 1995.

27. Liao W, Lin JX and Leonard WJ: IL-2 family cytokines: New insights into the complex roles of IL-2 as a broad regulator of T helper cell differentiation. Curr Opin Immunol 23: 598-604, 2011

28. Slingluff CL Jr: The present and future of peptide vaccines for cancer: Single or multiple, long or short, alone or in combination? Cancer J 17: 343-350, 2011.

29. Hallez S, Brulet JM, Vandooren C, Maudoux F, Thomas S Heinderickx M, Bollen A, Wattiez R and Jacquet A: Pre-clinical immunogenicity and anti-tumour efficacy of a deleted recombinant human papillomavirus type 16 E7 protein. Anticancer Res 24: 2265-2275, 2004.

30. Welters MJ, Filippov DV, van den Eeden SJ, Franken KL, Nouta J, Valentijn AR, van der Marel GA, Overkleeft HS, Lipford G, Offringa R, et al: Chemically synthesized protein as tumourspecific vaccine: Immunogenicity and efficacy of synthetic HPV16 E7 in the TC-1 mouse tumour model. Vaccine 23: 305-311, 2004.
31. Weis WI, Taylor ME and Drickamer K: The C-type lectin superfamily in the immune system. Immunol Rev 163: 19-34, 1998.

32. Royer PJ, Emara M, Yang C, Al-Ghouleh A, Tighe P, Jones N, Sewell HF, Shakib F, Martinez-Pomares L and Ghaemmaghami AM: The mannose receptor mediates the uptake of diverse native allergens by dendritic cells and determines allergen-induced $\mathrm{T}$ cell polarization through modulation of IDO activity. J Immunol 185: 1522-1531, 2010.

33. Hattori Y, Kawakami S, Suzuki S, Yamashita F and Hashida M: Enhancement of immune responses by DNA vaccination through targeted gene delivery using mannosylated cationic liposome formulations following intravenous administration in mice. Biochem Biophys Res Commun 317: 992-999, 2004.

34. Arigita C, Bevaart L, Everse LA, Koning GA, Hennink WE, Crommelin DJ, van de Winkel JG, van Vugt MJ, Kersten GF and Jiskoot W: Liposomal meningococcal B vaccination: Role of dendritic cell targeting in the development of a protective immune response. Infect Immun 71: 5210-5218, 2003.

35. Vaughan HA, Ho DW, Karanikas V, Sandrin MS, McKenzie IF and Pietersz GA: The immune response of mice and cynomolgus monkeys to macaque mucin 1-mannan. Vaccine 18: 3297-3309, 2000.

36. Toda S, Ishii N, Okada E, Kusakabe KI, Arai H, Hamajima K, Gorai I, Nishioka K and Okuda K: HIV-1-specific cell-mediated immune responses induced by DNA vaccination were enhanced by mannan-coated liposomes and inhibited by anti-interferongamma antibody. Immunology 92: 111-117, 1997. 